\title{
Low SMC1A protein expression predicts poor survival in acute myeloid leukemia
}

\author{
CLAUDIA HÖMME $^{1 *}$, UTZ KRUG $^{1 *}$, NICOLA TIDOW ${ }^{1}$, BERND SCHULTE $^{2}$, GABRIELE KÖHLER $^{2}$, \\ HUBERT SERVE ${ }^{4}$, HORST BÜRGER ${ }^{5}$, WOLFGANG E. BERDEL ${ }^{1}$, MARTIN DUGAS ${ }^{3}$, ACHIM HEINECKE ${ }^{3}$, \\ THOMAS BÜCHNER ${ }^{1}$, STEFFEN KOSCHMIEDER ${ }^{1}$ and CARSTEN MÜLLER-TIDOW ${ }^{1}$ \\ ${ }^{1}$ Department of Medicine A, University Hospital of Münster, Albert-Schweitzer-Str. 33, D-48149 Münster, \\ ${ }^{2}$ Department of Pathology, University Hospital of Münster, Domagkstr. 17, D-48149 Münster, \\ ${ }^{3}$ Department of Medical Informatics and Biomathematics, University Hospital of Münster, Domagkstr. 9, \\ D-48149 Münster; ${ }^{4}$ Department of Medicine II, University Hospital of Frankfurt, Theodor-Stern-Kai 7, \\ D-60590 Frankfurt am Main; ${ }^{5}$ Institute of Pathology, Husener Str. 46a, D-33098 Paderborn, Germany
}

Received March 2, 2010; Accepted April 15, 2010

DOI: $10.3892 /$ or_00000827

\begin{abstract}
Age is a strong adverse prognostic factor in acute myeloid leukemia. Little is known about the biology of acute myeloid leukemia in elderly patients. The aim of this study was to identify genes with age-dependent changes of expression in leukemic blasts and their relevance for the patient prognosis. Gene expression profiling was carried out by mRNA microarray analysis from blasts of 67 adult acute myeloid leukemia patients of different age (range, 17-80 years). Among the genes that correlated with age, PRPF4 and SMC1A were selected for protein expression studies on a tissue array containing bone marrow histologies of 135 patients with newly diagnosed AML of different ages. A significant correlation between mRNA expression levels and patient age was shown by 131 genes. Increasing age was associated with significantly decreased mRNA levels of SMC1A. On the protein level, expression of SMC1A was low or absent in 74 out of 116 acute myeloid leukemia specimens. Importantly, patients with low protein expression levels of SMC1A experienced significantly shortened event free (2.6 months versus 10.3 months, $\mathrm{p}=0.003)$ and overall survival (10.4 months versus 22.6 months, $\mathrm{p}=0.015)$. The $\mathrm{SMC1A}$ protein expression level remained a significant prognostic factor for event free survival $(\mathrm{p}=0.014)$ with a borderline significance for overall survival $(\mathrm{p}=0.066)$ in a multivariate analysis. SMC1A protein expression might
\end{abstract}

Correspondence to: Dr Utz Krug, Department of Medicine A, University Hospital of Münster, Albert-Schweitzer-Str. 33, D-48149 Münster, Germany

E-mail: utz.krug@ukmuenster.de

${ }^{*}$ Contributed equally

Key words: acute myeloid leukemia, prognostic factor, tissue microarray, immunohistochemistry, SMC1A play a role in the determination of the prognosis and might have possible implications in therapy decision in patients with acute myeloid leukemia.

\section{Introduction}

Acute myeloid leukemia (AML) is a genetically heterogeneous disease. Several prognostic factors have been identified such as age, sex, lactate dehydrogenase serum levels, recurrent structural (i.e. balanced translocations, inversions, deletions, insertions) or numerical (monosomy, trisomy) cytogenetic abnormalities (1), genetic mutations (i.e. mutational status of FLT3, NPM1, C/EBP $\alpha$, N-Ras, MLL, WT1) $(2,3)$, or changes in mRNA (i.e. ERG, BAALC, MN1, EVI1) (4-7) or protein (P-glycoprotein, lung-resistance protein) $(8,9)$ expression levels.

However, the identification of prognostic subgroups is a theme of growing complexity, for example a poor prognosis subgroup defined by c-kit mutations (10) or a loss of chromosome Y (11) can be demonstrated in the 'good' prognosis group of core binding factor leukemias in younger patients $<60$ years of age. These data argue for more detailed studies of risk factors in elderly patients with AML. However, most risk factors so far have been defined in patients aged $<60$ years.

Despite advances in the therapy of AML over the last decades, the prognosis of AML in patients $>60$ years of age is still dismal (12). Adverse prognostic factors such as an adverse cytogenetic profile (12) or the expression of P-glycoprotein occur more frequently in AML blasts of elderly patients $(13,14)$. Recently, we demonstrated age as the most important independent risk factor for the prognosis among 2734 patients with AML in the multicenter German AML Co-operative Group (AMLCG) study AMLCG1999, and this remained highly significant as an independent risk factor in a multivariate analysis $(15,16)$. Many studies have demonstrated a decrease of good-risk cytogenetics with increasing age, suggesting that AML in elderly patients differs biologically from AML in younger patients (17). 
However, the relevant biological mechanisms of the poor prognosis of AML in older patients are unknown.

Therefore, the aim of this study was to detect age-dependent changes in AML by a genome-wide gene expression profile. We were able to identify a variety of genes with agedependent changes in mRNA expression, and in addition we identified a new prognostic protein marker, SMC1A, in acute myeloid leukemia.

\section{Materials and methods}

Patient samples. For mRNA expression analysis, EDTAanticoagulated bone marrow aspirates were obtained at diagnosis from 67 adult AML patients of different age (range, 17-80 years). For tissue microarray preparation, bone marrow trephines were obtained from 135 patients with newly diagnosed AML. Bone marrow trephines were formalin-fixed and paraffin-embedded according to standard procedures. Written informed consent was obtained from all patients and the study was approved by the local Institutional Review Board (Ethikkomission der WWU Münster) and only patients treated at the University Hospital of Muenster were included in this study. The studies were in compliance with all applicable national and local ethics guidelines.

RNA isolation. AML blasts were isolated from EDTAanticoagulated bone marrow aspirates at diagnosis by Ficollhypaque density centrifugation according to standard procedures. Total RNA was isolated using TRIzol ${ }^{\circledR}$-Reagent (Invitrogen, Karlsruhe, Germany) according to the manufacturer's protocol.

mRNA expression arrays. Microarray expression analysis was performed using the ABI 1700 microarray system accoding to the manufacturer's protocol (18). Two micrograms of total RNA were reverse-transcribed and second-strand synthesis was performed. Digoxigenin (DIG)UTP was included in the IVT-reaction to produce DIGlabeled cRNA that after fragmentation was hybridized to the Genome Survey Expression Arrays covering 31,700 oligonucleotide probes representing 29,098 individual genes (ABI). Detection was performed by a chemoluminescent reaction with high sensitivity. Initial analyses were performed with the 1700 chemiluminescent microarray analyser (ABI).

Preparation of tissue microarrays. Formalin-fixed and paraffin-embedded tissue of 135 patients with newly diagnosed AML were used for the production of a tissue microarray as previously described (19). A diagnostic Giemsa-stained section served as control to enable the definition of areas with the highest amount of blast cells. Two punches were arrayed per sample. The spot diameter was $0.6 \mathrm{~mm}$, and the distance between the spots was $1 \mathrm{~mm}$.

Array immunohistochemistry and quantification. Tissue sections $(4-\mu \mathrm{m})$ were mounted on SuperFrost/Plus slides and dewaxed in xylene. The sections were autoclaved in $10 \mathrm{mM}$ citrate buffer $\mathrm{pH} 6.0\left(10 \mathrm{~min}, 120^{\circ} \mathrm{C}\right)$. After washing in PBS, sections were incubated with the primary antibodies (PRPF4, Santa Cruz Biotechnology, T-24, dilution 1:100;

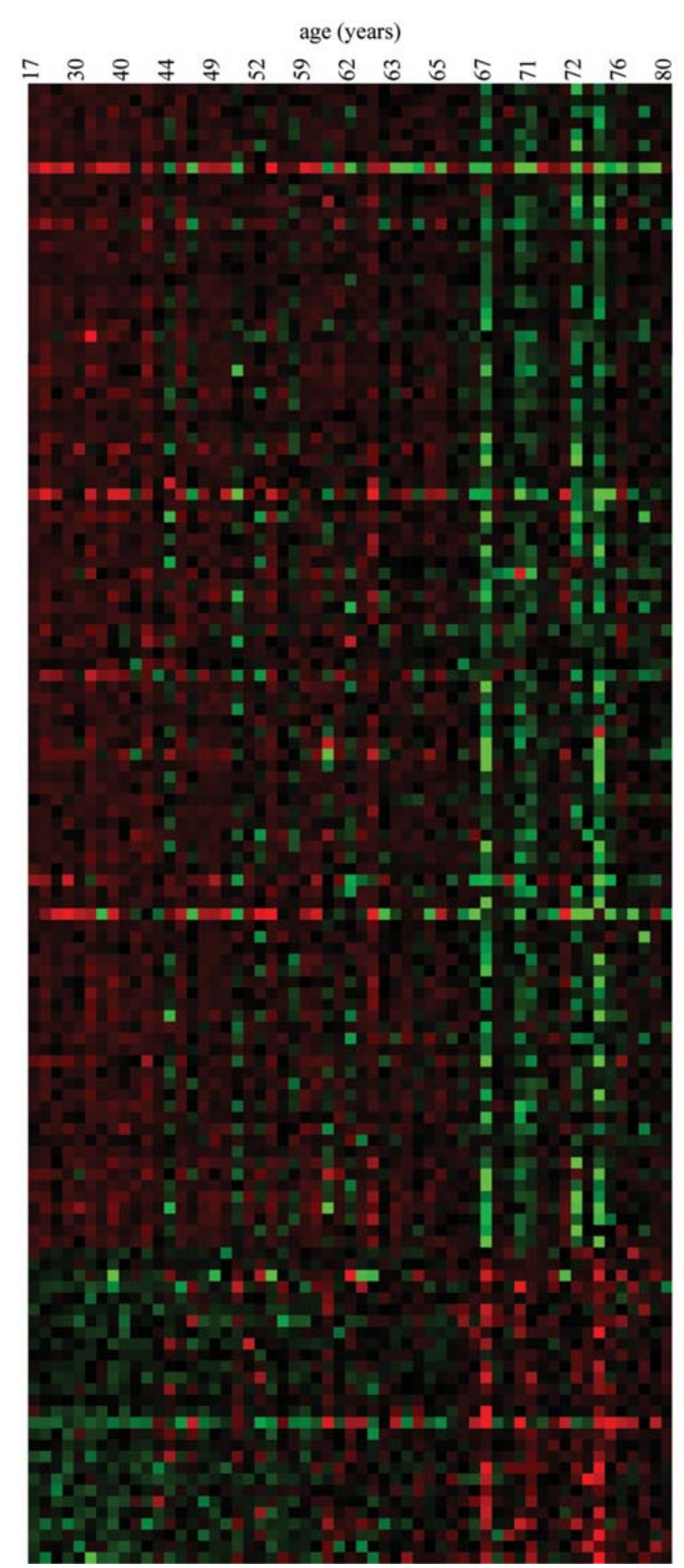

Figure 1. Heatmap of mRNA expression levels with regard to patient age. Genes correlating with age were identified and analyzed in a hierarchical cluster analysis. Patients are grouped on the heatmap according to age. Green, expression level lower than the median for this gene; red, expression level higher than the median for this gene. The gene names can be identified in Table I, where the genes are displayed the same order as in the Figure.

SMC1A, Atlas Antibodies, dilution 1:50). Detection was performed by the use of a 3,3'-diaminobenzidine detection kit (ER Ventana), and with the APAAP using a monoclonal APAAP complex (1:100 in RPM-1640, $60 \mathrm{~min}$ in room temperature; Dako). Quantification of immunohistochemical staining in AML blasts by a pathologist was performed according to conventional standards (Dako score). 

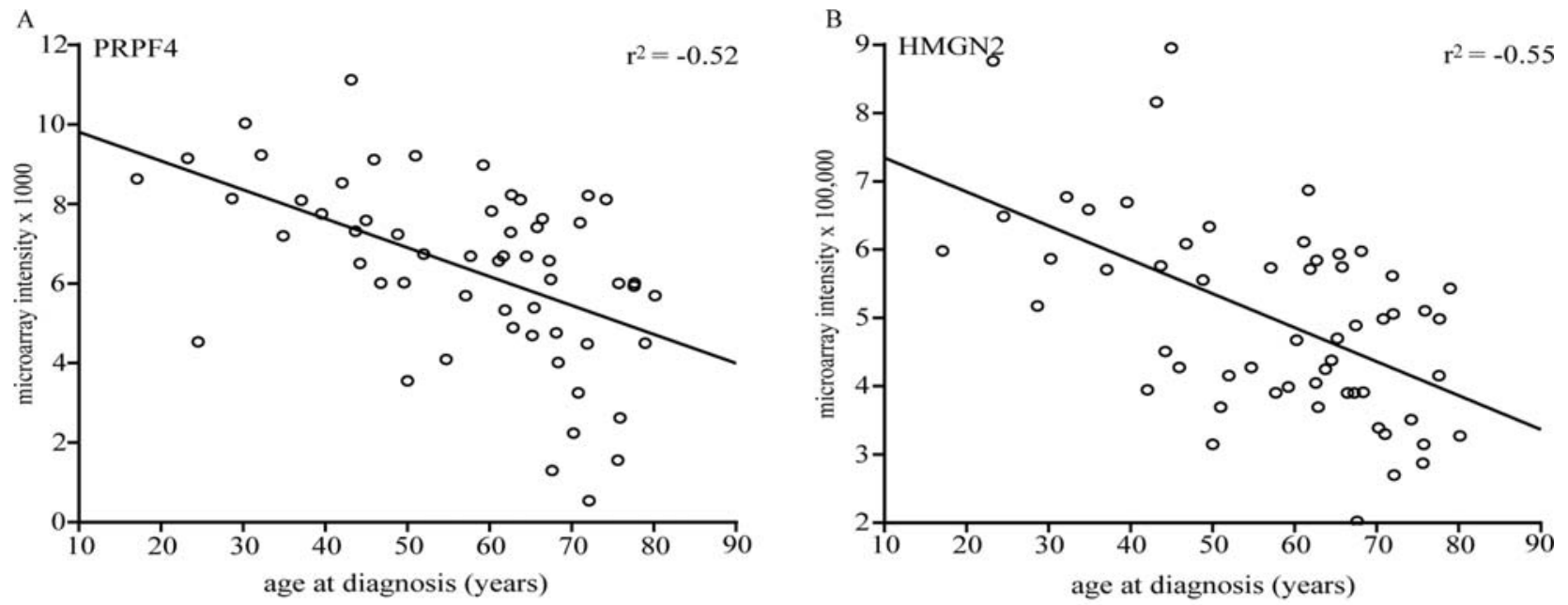

$\mathrm{C}$

D
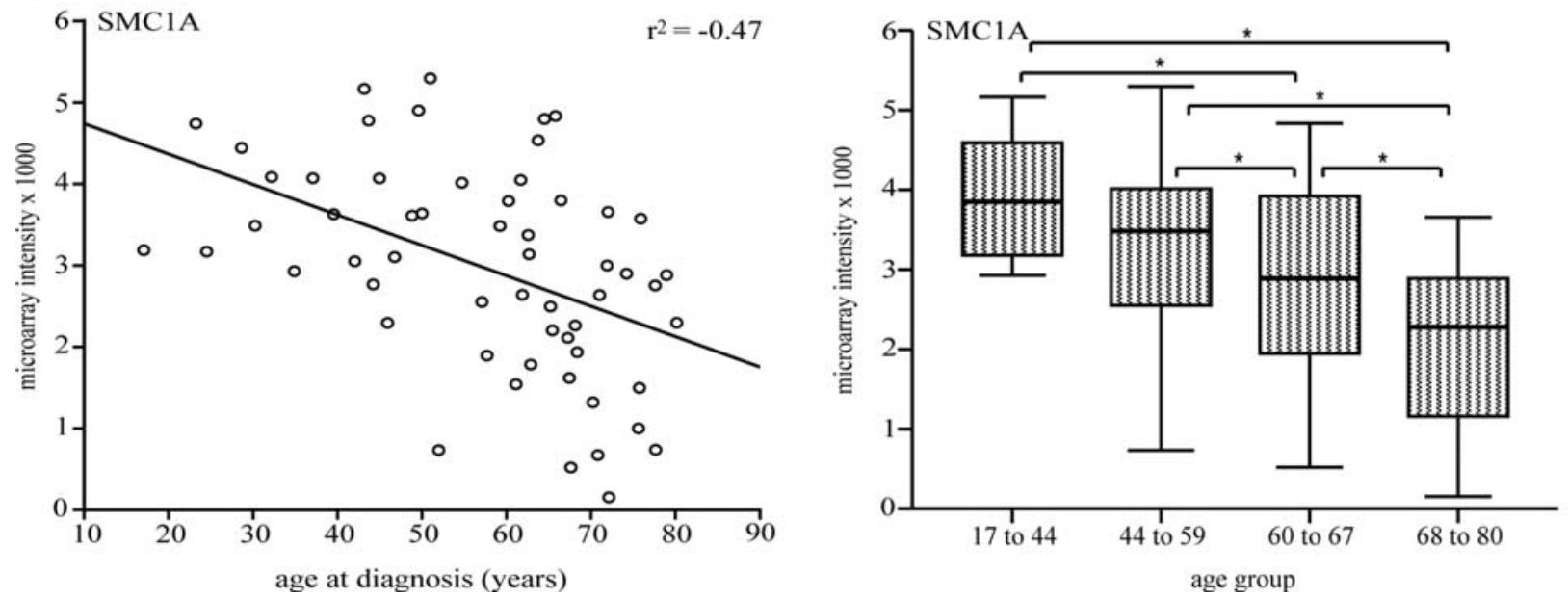

Figure 2. Age-dependent mRNA expression changes in AML for three representative genes. (A) PRPF4, (B) HMGN2, (C and D) SMC1A. " $\mathrm{p}<0.05$.

Statistical analysis. Microarray data were analyzed by BRB Array tools. A correlation analysis was performed between expression and age at a significance level of $\mathrm{p}<0.001$. Statistical analysis of protein expression was performed using the Statistical Package for the social Sciences (SPSS), version SPSS V17.0 for Windows. Survival analysis was performed by Kaplan-Meier analysis with a log-rank test for significance and a significance level of $\alpha=0.05$. Statistical analysis of clinical parameters were performed with either Wilcoxon-test or $\chi^{2}$-test for homogeneity where applicable, both with a significance level of $\alpha=0.05$. Multivariate analysis was performed by multivariate Cox regression with a significance level of $\alpha=0.05$.

\section{Results}

Identification of age-dependent gene expression by microarray expression analysis. To start to unravel the molecular basis for the prognostic effect of age in AML we performed microarray expression analyses in AML patients. In the mRNA microarray analysis, 131 genes showed a significant correlation between the expression level and the patient age at the time of diagnosis; 103 displayed a negative and only 28 a positive correlation (Fig. 1; Table I). Three examples of age dependent genes are shown (Fig. 2). HMGN2 (high- mobility group nucleosomal binding domain 2), PRPF4 (pre-mRNA processing factor 4 homolog) and SMC1A (structural maintenance of chromosomes 1A) microarray expression results negatively correlated with the patient age at diagnosis.

PRPF4 and SMC1A protein expression analysis and correlation with $m R N A$ expression. Association of mRNA level changes with clinical parameters might be an indication of regulatory phenomenon rather than a direct evidence for biologically relevant genes. We therefore used an AML tissue microarray to identify whether the altered mRNA expression levels were associated with patient survival based on protein expression data. PRPF4 and SMC1A were further analyzed by immunohistochemistry due to its known function during mitosis and due to antibody availability. Assessment of the PRPF4 and the SMC1A protein expression was sufficient in 115 and 116 out of 135 patient samples, respectively. For the remaining 20 and 19 samples, an insufficient number of AML blasts were spotted on the tissue microarray. One representative picture of a negative (expression level 0) and a positive (expression level 3) expression of SMC1A is presented (Fig. 3).

Protein levels of either PRPF4 or SMC1A protein detected by immunohistochemistry on tissue microarrays did not 
Table I. Age-dependent changes in mRNA expression levels.

\begin{tabular}{|c|c|c|c|c|c|}
\hline $\begin{array}{l}\text { Unique } \\
\text { id }\end{array}$ & $\begin{array}{l}\text { Correlation } \\
\text { coefficient }\end{array}$ & Description & Clone & $\begin{array}{c}\text { GeneBank } \\
\text { accession no. }\end{array}$ & Gene symbol \\
\hline 109690 & -0.553 & $\begin{array}{l}\text { PRP4 pre-mRNA processing factor } 4 \\
\text { homolog (yeast) }\end{array}$ & 86685231 & NM_004697 & PRPF4 \\
\hline 146792 & -0.542 & $\begin{array}{l}\text { High-mobility group nucleosomal binding } \\
\text { domain } 2\end{array}$ & & NM_005517 & HMGN2 \\
\hline 110983 & -0.531 & Chromosome 19 open reading frame 28 & 3474869 & NM_174983 & C19orf28 \\
\hline 197989 & -0.523 & Mitochondrial ribosomal protein L18 & 160857025 & NM_014161 & MRPL18 \\
\hline 101755 & -0.52 & & 107999084 & & \\
\hline 165131 & -0.513 & $\begin{array}{l}\text { SMC1 structural maintenance of } \\
\text { chromosomes 1-like } 1 \text { (yeast) }\end{array}$ & 57232625 & NM_006306 & SMC1L1 \\
\hline 236541 & -0.511 & & & AL391416 & \\
\hline 126222 & -0.51 & Hypothetical protein FLJ32942 & 54501444 & AK057504 & FLJ32942 \\
\hline 212663 & -0.508 & & 111502434 & & \\
\hline 173693 & -0.506 & HSPC244 & 58495163 & NM_016499 & MGC:13379 \\
\hline 197251 & -0.505 & $\begin{array}{l}\text { Solute carrier family } 1 \text { (neutral amino acid } \\
\text { transporter), member } 5\end{array}$ & 44082394 & NM_005628 & SLC1A5 \\
\hline 109395 & -0.505 & & 57264512 & & \\
\hline 169499 & -0.503 & $\begin{array}{l}\text { Anillin, actin binding protein (scraps } \\
\text { homolog, Drosophila) }\end{array}$ & 36413980 & NM_018685 & ANLN \\
\hline 128737 & -0.503 & & & & \\
\hline 135002 & -0.502 & $\begin{array}{l}\text { High-mobility group nucleosomal } 2 \\
\text { binding domain }\end{array}$ & 5599960 & NM_005517 & HMGN2 \\
\hline 183246 & -0.501 & $\begin{array}{l}\text { Heterogeneous nuclear ribonucleoprotein } \\
\mathrm{C}(\mathrm{C} 1 / \mathrm{C} 2)\end{array}$ & 1538809 & NM_031314 & HNRPC \\
\hline 139758 & -0.5 & & 52964773 & & \\
\hline 185984 & -0.498 & KIAA0261 & 82193861 & NM_015045 & KIAA0261 \\
\hline 140552 & -0.498 & & 45407426 & & \\
\hline 106136 & -0.497 & & 3569763 & & \\
\hline 143238 & -0.491 & $\begin{array}{l}\text { CSE1 chromosome segregation 1-like } \\
\text { (yeast) }\end{array}$ & 44367400 & NM_001316 & CSE1L \\
\hline 153495 & -0.49 & & 44104207 & & \\
\hline 134014 & -0.488 & $\begin{array}{l}\text { Sialyltransferase } 6(\mathrm{~N} \text {-acetyllacosaminide } \\
\alpha 2,3 \text {-sialyltransferase) }\end{array}$ & 42455024 & NM_174972 & SIAT6 \\
\hline 188782 & -0.485 & & 79209516 & & \\
\hline 130790 & -0.485 & & 13757670 & & \\
\hline 143413 & -0.485 & DUF729 domain containing 1 & 137292608 & NM_138419 & DUFD1 \\
\hline 125707 & -0.48 & & 79836088 & & \\
\hline 101962 & -0.474 & & 134948938 & & \\
\hline 125645 & -0.473 & & 80451333 & & \\
\hline 155269 & -0.471 & $\begin{array}{l}\text { ATP synthase, } \mathrm{H}^{+} \text {transporting, mito- } \\
\text { chondrial } \mathrm{F} 1 \text { complex, } ß \text { polypeptide }\end{array}$ & 56683667 & NM_001686 & ATP5B \\
\hline 140313 & -0.47 & $\begin{array}{l}\text { High-mobility group nucleosomal } 2 \\
\text { binding domain }\end{array}$ & 7664137 & NM_005517 & HMGN2 \\
\hline 100130 & -0.469 & & 25910808 & Z83840 & \\
\hline 191627 & -0.469 & Nuclear receptor coactivator 4 & 42865708 & NM_005437 & NCOA4 \\
\hline 208076 & -0.465 & Transportin 3 & 123390730 & NM_012470 & TNPO3 \\
\hline 191104 & -0.465 & $\begin{array}{l}\text { High-mobility group nucleosomal binding } \\
\text { domain } 2\end{array}$ & 25195509 & NM_005517 & HMGN2 \\
\hline 211546 & -0.464 & $\begin{array}{l}\text { Procollagen-proline, 2-oxoglutarate 4- } \\
\text { dioxygenase (proline 4-hydroxylase), } \\
\text { ß polypeptide (protein disulfide isomerase; } \\
\text { thyroid hormone binding protein p55) }\end{array}$ & 76400747 & NM_000918 & $\mathrm{P} 4 \mathrm{HB}$ \\
\hline
\end{tabular}


Table I. Continued.

\begin{tabular}{|c|c|c|c|c|c|}
\hline $\begin{array}{l}\text { Unique } \\
\text { id }\end{array}$ & $\begin{array}{l}\text { Correlation } \\
\text { coefficient }\end{array}$ & Description & Clone & $\begin{array}{l}\text { GeneBank } \\
\text { accession no. }\end{array}$ & Gene symbol \\
\hline 166551 & -0.464 & & 1248647 & X55989 & \\
\hline 100220 & -0.463 & & & & \\
\hline 199549 & -0.462 & $\begin{array}{l}\text { CDC91 cell division cycle 91-like } 1 \\
\text { (S. cerevisiae) }\end{array}$ & 29897423 & NM_080476 & CDC91L1 \\
\hline 234027 & -0.462 & & 13859989 & & \\
\hline 138129 & -0.461 & $\begin{array}{l}\text { Procollagen-lysine, 2-oxoglutarate 5- } \\
\text { dioxygenase } 3\end{array}$ & 95858416 & NM_001084 & PLOD3 \\
\hline 137614 & -0.461 & Hypothetical protein MGC3234 & 935474 & NM_023947 & MGC3234 \\
\hline 141237 & -0.461 & $\begin{array}{l}\text { Protein phosphatase } 2 \text { (formerly } 2 \mathrm{~A}) \text {, } \\
\text { regulatory subunit A (PR 65), } \beta \text { isoform }\end{array}$ & 108749256 & NM_181699 & PPP2R1B \\
\hline 103896 & -0.461 & Zinc finger protein 239 & 40055428 & NM_005674 & ZNF239 \\
\hline 131916 & -0.458 & & 70596609 & & \\
\hline 107045 & -0.457 & Chromosome 10 open reading frame 69 & 95647794 & NM_006459 & C10orf69 \\
\hline 179303 & -0.456 & Polymerase (DNA directed), $\alpha$ & 28834934 & NM_016937 & POLA \\
\hline 235868 & -0.456 & Glyceraldehyde-3-phosphate dehydrogenase & 8260480 & NM_002046 & GAPD \\
\hline 197341 & -0.453 & Polo-like kinase 1 (Drosophila) & 22468349 & NM_005030 & PLK1 \\
\hline 198244 & -0.453 & $\begin{array}{l}\text { Nuclear factor of kappa light polypeptide } \\
\text { gene enhancer in B-cells inhibitor-like } 2\end{array}$ & 141828520 & NM_013432 & NFKBIL2 \\
\hline 104988 & -0.453 & $\begin{array}{l}\text { COP9 constitutive photomorphogenic } \\
\text { homolog subunit } 3 \text { (Arabidopsis) }\end{array}$ & 18089553 & NM_003653 & COPS3 \\
\hline 212751 & -0.452 & & 26090623 & & \\
\hline 162809 & -0.451 & Ribokinase & 27849146 & NM_022128 & RBKS \\
\hline 186772 & -0.451 & HSPC154 protein & 68680276 & NM_014177 & HSPC154 \\
\hline 202943 & -0.451 & & 151204786 & & \\
\hline 169279 & -0.451 & $\begin{array}{l}\text { AT rich interactive domain } 3 \mathrm{~A} \\
\text { (BRIGHT-like) }\end{array}$ & 861394 & NM_005224 & ARID3A \\
\hline 217900 & -0.449 & Tumor rejection antigen (gp96) 1 & & NM_003299 & TRA1 \\
\hline 129518 & -0.449 & $\begin{array}{l}\text { Putative endoplasmic reticulum multispan } \\
\text { transmembrane protein }\end{array}$ & 53092105 & NM_052859 & RFT1 \\
\hline 157917 & -0.447 & Serine/threonine kinase 6 & 51683782 & NM_003600 & STK6 \\
\hline 208898 & -0.447 & & 1864863 & NM_014867 & \\
\hline 129633 & -0.446 & & & AK023204 & \\
\hline 144711 & -0.445 & Mitochondrial ribosomal protein L27 & 44906512 & NM_016504 & MRPL27 \\
\hline 105423 & -0.445 & $\mathrm{H} 2 \mathrm{~A}$ histone family, member $\mathrm{Z}$ & 98166661 & NM_002106 & H2AFZ \\
\hline 176188 & -0.444 & Golgi autoantigen, golgin subfamily a, 2 & 101669854 & NM_004486 & GOLGA2 \\
\hline 236939 & -0.442 & & 81080616 & & \\
\hline 111979 & -0.441 & DKFZP434I116 protein & 91686315 & NM_015496 & DKFZP434I116 \\
\hline 163631 & -0.441 & & 73658572 & & \\
\hline 174829 & -0.441 & $\begin{array}{l}\text { Phosphatidylinositol-4-phosphate 5-kinase, } \\
\text { type I, } \alpha\end{array}$ & 124286312 & NM_003557 & PIP5K1A \\
\hline 151175 & -0.441 & & 45121345 & & \\
\hline 150309 & -0.441 & Chromosome 10 open reading frame 7 & 12167391 & NM_006023 & C10orf7 \\
\hline 104729 & -0.44 & Similar to common salivary protein 1 & 3080178 & NM_145252 & LOC124220 \\
\hline 123864 & -0.44 & Hypothetical protein $\mathrm{H} 17$ & 123279476 & NM_017547 & H17 \\
\hline 160632 & -0.44 & Signal recognition particle $68 \mathrm{kDa}$ & 70625229 & NM_014230 & SRP68 \\
\hline 134187 & -0.438 & $\begin{array}{l}\text { Membrane-spanning 4-domains, subfamily A, } \\
\text { member } 3 \text { (hematopoietic cell-specific) }\end{array}$ & 57188481 & NM_006138 & MS4A3 \\
\hline 211285 & -0.438 & & 65806540 & & \\
\hline 174651 & -0.438 & Karyopherin $\alpha 1$ (importin $\alpha$ 5) & 120553552 & NM_002264 & KPNA1 \\
\hline 223004 & -0.438 & Zinc finger protein 561 & 9615731 & NM_152289 & ZNF561 \\
\hline 209604 & -0.438 & & 178067648 & & \\
\hline
\end{tabular}


Table I. Continued.

\begin{tabular}{|c|c|c|c|c|c|}
\hline $\begin{array}{l}\text { Unique } \\
\text { id }\end{array}$ & $\begin{array}{l}\text { Correlation } \\
\text { coefficient }\end{array}$ & Description & Clone & $\begin{array}{c}\text { GeneBank } \\
\text { accession no. }\end{array}$ & Gene symbol \\
\hline 200565 & -0.437 & F-box protein 22 & 53122127 & NM_012170 & FBXO22 \\
\hline 110781 & -0.436 & ATPase type 13A & 19660081 & NM_020410 & ATP13A \\
\hline 178526 & -0.436 & $\begin{array}{l}\text { Thioredoxin domain containing } 7 \\
\text { (protein disulfide isomerase) }\end{array}$ & 10838664 & NM_005742 & TXNDC7 \\
\hline 157593 & -0.436 & Lectin, mannose-binding 2 & 171722402 & NM_006816 & LMAN2 \\
\hline 118987 & -0.436 & $\begin{array}{l}\text { NADH dehydrogenase (ubiquinone) } 1 \\
\alpha \text { subcomplex, assembly factor } 1\end{array}$ & 18447520 & NM_016013 & NDUFAF1 \\
\hline 197221 & -0.436 & $\begin{array}{l}\text { Methylene tetrahydrofolate dehydrogenase } \\
\left(\mathrm{NAD}^{+} \text {dependent }\right), \text { methenyltetrahydrofolate } \\
\text { cyclohydrolase }\end{array}$ & 74256918 & NM_006636 & MTHFD2 \\
\hline 116667 & -0.435 & Calpain small subunit 2 & 40114805 & NM_032330 & CAPNS2 \\
\hline 110562 & -0.435 & $\begin{array}{l}\text { Processing of precursor } 4 \text {, ribonuclease } \\
\text { P/MRP subunit (S. cerevisiae) }\end{array}$ & 26795014 & NM_006627 & POP4 \\
\hline 115589 & -0.434 & $\begin{array}{l}\text { MCM4 minichromosome maintenance } \\
\text { deficient } 4 \text { (S. cerevisiae) }\end{array}$ & 44858253 & NM_005914 & MCM4 \\
\hline 192701 & -0.434 & $\begin{array}{l}\text { Ret proto-oncogene (multiple endocrine } \\
\text { neoplasia and medullary thyroid carcinoma } 1 \text {, } \\
\text { Hirschsprung disease) }\end{array}$ & 30476899 & NM_020629 & RET \\
\hline 148733 & -0.434 & & & ВC020307 & \\
\hline 135285 & -0.433 & $\begin{array}{l}\text { Integral membrane protein } 1 ; \text { CHK1 } \\
\text { checkpoint homolog }(\mathrm{S} . \text { pombe })\end{array}$ & 122623025 & NM_001274 & ITM1; CHEK1 \\
\hline 186340 & -0.433 & DKFZP566O084 protein & 21447444 & NM_015510 & DKFZp5660084 \\
\hline 210481 & -0.433 & $\begin{array}{l}\text { Golgi associated, } \gamma \text { adaptin ear } \\
\text { containing, ARF binding protein } 2\end{array}$ & 22252488 & NM_138640 & GGA2 \\
\hline 229412 & -0.432 & & 40788561 & & \\
\hline 199189 & -0.432 & & 136896507 & & \\
\hline 236093 & -0.431 & $\begin{array}{l}\text { Eukaryotic translation initiation factor } 4 \mathrm{~A} \text {, } \\
\text { isoform } 1\end{array}$ & 7502345 & NM_001416 & EIF4A1 \\
\hline 175324 & -0.43 & Glyceraldehyde-3-phosphate dehydrogenase & 8260480 & NM_002046 & GAPD \\
\hline 160577 & -0.43 & Kinesin family member 23 & 46595415 & NM_138555 & KIF23 \\
\hline 212857 & -0.43 & Bifunctional apoptosis regulator & 14903442 & NM_016561 & BFAR \\
\hline 213392 & -0.429 & & 118332014 & & \\
\hline 186923 & -0.429 & $\begin{array}{l}\text { 3'-phosphoadenosine 5'-phosphosulfate } \\
\text { synthase } 1\end{array}$ & 105829640 & NM_005443 & PAPSS1 \\
\hline 184872 & -0.429 & $\begin{array}{l}\text { Dystonia 1, torsion (autosomal dominant; } \\
\text { torsin A) }\end{array}$ & 103225965 & NM_000113 & DYT1 \\
\hline 105868 & -0.428 & $\begin{array}{l}\text { Translocase of outer mitochondrial } \\
\text { membrane } 34\end{array}$ & 40279403 & NM_006809 & TOMM34 \\
\hline 143257 & -0.428 & $\begin{array}{l}\text { Dolichyl-diphosphooligosaccharide-protein } \\
\text { glycosyltransferase }\end{array}$ & 19301250 & NM_005216 & DDOST \\
\hline 111860 & 0.431 & $\begin{array}{l}\text { Solute carrier family } 25 \text { (mitochondrial } \\
\text { carrier; ornithine transporter) member } 2\end{array}$ & 136759020 & NM_031947 & SLC25A2 \\
\hline 212706 & 0.433 & & 38909003 & ВC039000 & \\
\hline 133654 & 0.434 & Tumor protein p73 & 2823529 & NM_005427 & TP73 \\
\hline 108888 & 0.435 & Kinesin family member $21 \mathrm{~A}$ & 38491505 & NM_017641 & KIF21A \\
\hline 148924 & 0.436 & Hypothetical protein MGC10500 & 72687482 & NM_031477 & MGC10500 \\
\hline 194902 & 0.438 & Chemokine (C-C motif) ligand 22 & 41892711 & NM_002990 & CCL22 \\
\hline 145792 & 0.442 & & 51330963 & ВC041467 & \\
\hline 234743 & 0.443 & chr3 synaptotagmin & 136578332 & NM_031913 & CHR3SYT \\
\hline 212352 & 0.444 & & 246294 & AX657520 & \\
\hline 116672 & 0.445 & Cryptochrome 2 (photolyase-like) & 46016602 & NM_021117 & CRY2 \\
\hline
\end{tabular}


Table I. Continued.

\begin{tabular}{|c|c|c|c|c|c|}
\hline $\begin{array}{l}\text { Unique } \\
\text { id }\end{array}$ & $\begin{array}{l}\text { Correlation } \\
\text { coefficient }\end{array}$ & Description & Clone & $\begin{array}{c}\text { GeneBank } \\
\text { accession no. }\end{array}$ & Gene symbol \\
\hline 103227 & 0.446 & Cytosolic acetyl-CoA hydrolase & 76530115 & NM_130767 & CACH-1 \\
\hline 228159 & 0.449 & & 23232740 & BX647251 & \\
\hline 175816 & 0.452 & $\begin{array}{l}\text { Hypothetical protein FLJ25070; amylase, } \\
\alpha \text { 2B; pancreatic; amylase, } \alpha 2 \mathrm{~A} \text {; pancreatic; } \\
\text { amylase, } \alpha \text { 1A; salivary }\end{array}$ & 102260708 & NM_000699 & $\begin{array}{l}\text { FLJ25070; } \\
\text { AMY2B; } \\
\text { AMY2A; } \\
\text { AMY1A }\end{array}$ \\
\hline 115606 & 0.453 & & 52351877 & BC057822 & \\
\hline 231470 & 0.454 & & 8763111 & BC038195 & \\
\hline 124737 & 0.46 & PRKC, apoptosis, WT1, regulator & 79645370 & NM_002583 & PAWR \\
\hline 208153 & 0.465 & $\begin{array}{l}\text { Hypothetical protein FLJ25070; amylase, } \\
\alpha \text { 2B; pancreatic; amylase, } \alpha 2 \mathrm{~A} \text {; pancreatic; } \\
\text { amylase, } \alpha \text { 1A; salivary }\end{array}$ & 102260708 & NM_017619 & $\begin{array}{l}\text { FLJ25070; } \\
\text { AMY2B; } \\
\text { AMY2A; } \\
\text { AMY1A }\end{array}$ \\
\hline 123083 & 0.466 & Chromosome 16 open reading frame 7 & 74837974 & NM_004913 & C16orf7 \\
\hline 141785 & 0.468 & Interleukin 11 receptor, $\alpha$ & 34582169 & NM_004512 & IL11RA \\
\hline 199475 & 0.475 & & 133220558 & ВC058028 & \\
\hline 179061 & 0.476 & Fetuin B & 184794512 & NM_014375 & FETUB \\
\hline 164097 & 0.48 & Hypothetical protein MGC34732 & 82875751 & NM_173556 & MGC34732 \\
\hline 209982 & 0.485 & Hypothetical protein MGC34728 & 124693182 & NM_152533 & MGC34728 \\
\hline 137266 & 0.498 & Protease inhibitor 16 & 38471077 & NM_153370 & PI16 \\
\hline 194177 & 0.503 & Hypothetical protein FLJ21159 & 153598959 & NM_024826 & FLJ21159 \\
\hline 166432 & 0.503 & & & ВC020998 & \\
\hline 160157 & 0.524 & & 58498648 & & \\
\hline 116936 & 0.554 & & 86943902 & NM_014883 & \\
\hline
\end{tabular}

Changes in mRNA expression were measured by microarray spot intensity. Genes are displayed in the same order as in Fig. 1. Sequences of the oligonucleotides spotted on the array can be obtained by the corresponding author.

correlate with age (data not shown). Both mRNA and protein expression data were available for 19 patients each for PRPF4 and SMC1A. In this subgroup, also no close correlation was observed between PRPF4 mRNA and protein expression or between SMC1A mRNA and protein expression (data not shown). These findings suggest that protein levels of the PRPF4 and SMC1A protein are regulated on several levels with mRNA expression levels being only one of them.

Loss of SMC1A protein expression indicates a poor prognosis in $A M L$. Immunohistochemistry for SMC1A showed only weak or absent expression in 74 patients. Moderate to high expression was observed in 42 patients. Similar to mRNA levels, patients with low SMC1A expression were slightly older (median 62 years) than patients with moderate to high expression (median 59 years). However, in contrast to the mRNA expression data, this difference was not statistically significant (Table II). No correlation was observed between SMC1A protein expression and the presence of a complexaberrant karyotype or between SMC1A protein expression and a normal karyotype versus the presence of any chromosomal abnormalities (data not shown).
A moderate to high SMC1A protein expression (expression level $>1+$ ) was associated with a significantly improved event free survival as well as overall survival (Fig. 4). Median overall survival was 22.6 versus 10.4 months $(\mathrm{p}=0.015)$ and median event free survival was 10.3 versus 2.6 months $(\mathrm{p}=0.003)$ for SMC1A moderate or high expression versus SMC1A low or absent expression, respective.

The patient characteristics of the 116 patients with low or undetectable $\left(\leq 1^{+}\right)$versus moderate or high $\left(>1^{+}\right)$SMC1A protein expression did not reveal significant differences regarding sex, age, FAB type, cytogenetic risk group, or an intensive versus a non-intensive treatment strategy (Table II). For this patient cohort, younger age at diagnosis ( $<60$ vs. $\geq 60$ years), female sex, de novo versus secondary leukemia, and low lactate dehydrogenase (LDH) serum level at diagnosis ( $\leq 700$ vs. $>700 \mathrm{U} / 1$ ) correlated with a significant better EFS in a univariate analysis in addition to moderate or high SMC1A protein level, whereas the cytogenetic risk group (low, intermediate or high) or white blood cell count at diagnosis $(\leq 20,000$ vs. $>20,000 / \mu 1)$ did not show a significant correlation with EFS. Out of this risk factors age, LDH serum level and SMC1A protein expression remained significant 


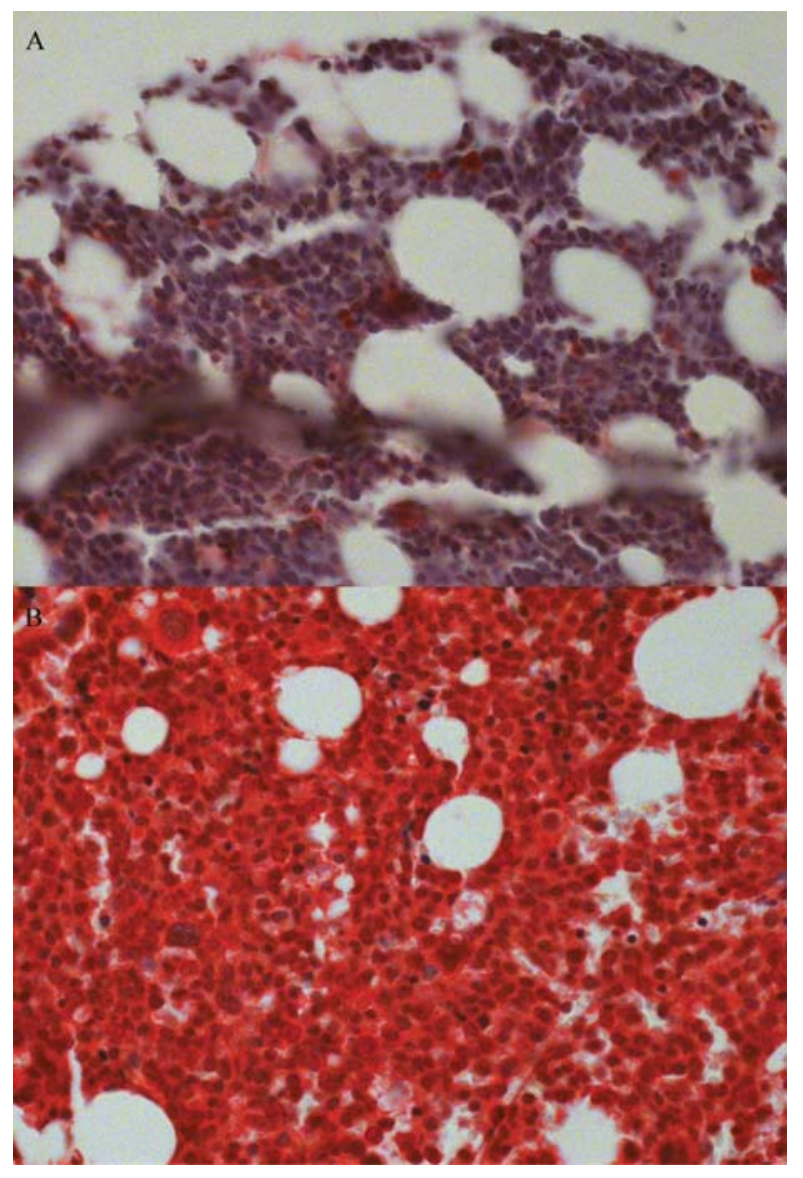

Figure 3. Immunohistochemistry staining of SMC1A on an AML tissue microarray. A tissue microarray containing AML bone marrow and control specimens was utilized to analyze protein expression of SMC1A. Images were acquired using a Zeiss Axio Imager M1 at a magnification x400. Examples for positive and negative expression are depicted. (A) Negative staining (Dako score 0): $<10 \%$ of the blast cells are positive for SMC1A (B) Strong positive staining (Dako score $3+$ ). The majority of blasts show a strong cytoplasmatic signal for SMC1A.

in a multivariate Cox-regression analysis (Table III). For overall survival, younger age, female gender and low LDH serum levels showed a significant positive correlation in addition to moderate or high SMC1A protein expression in a univariate analysis. Only gender remained significant in a multivariate analysis, with borderline significance $(\mathrm{p}=0.066$, two-tailed) for SMC1A protein expression (Table III).

Since only two out of 115 evaluable samples showed a moderate PRPF4 expression, no further analysis of PRPF4 expression with clinical parameters was performed.

\section{Discussion}

In this study, we identified age-related changes in gene expression of AML blasts. In our analysis, 131 genes showed a significant correlation of the mRNA expression level with the patient age at diagnosis. For none of these genes has an age-dependent expression been described previously in AML. Interestingly, at the high stringency level of analysis that we utilized, we could not detect the previously described age-dependent increase of mRNA expression of the gene ABCB1 (MDR1) $(13,14)$.
Table II. Clinical characteristics of the patients analyzed for SMC1A protein expression.

\begin{tabular}{|c|c|c|c|}
\hline SMC expression level & $\begin{array}{c}\text { Absent/ } \\
\text { low }\end{array}$ & $\begin{array}{l}\text { Moderate/ } \\
\text { high }\end{array}$ & P-value \\
\hline No. of patients (n) & 74 & 42 & \\
\hline $\begin{array}{l}\text { Age at diagnosis } \\
\text { (years), median (range) }\end{array}$ & $62(18-85)$ & $59(21-82)$ & 0.78 \\
\hline Gender & & & 0.90 \\
\hline Male, n (\%) & $44(59)$ & $22(52)$ & \\
\hline Female, n (\%) & $30(41)$ & $20(48)$ & \\
\hline FAB type & & & 0.93 \\
\hline $\mathrm{M} 0, \mathrm{n}(\%)$ & $0(0)$ & $1(2)$ & \\
\hline $\mathrm{M} 1, \mathrm{n}(\%)$ & $8(11)$ & $6(14)$ & \\
\hline $\mathrm{M} 2, \mathrm{n}(\%)$ & $13(18)$ & $11(26)$ & \\
\hline $\mathrm{M} 3, \mathrm{n}(\%)$ & $2(3)$ & $0(0)$ & \\
\hline $\mathrm{M} 4, \mathrm{n}(\%)$ & $17(23)$ & $11(26)$ & \\
\hline M5, n (\%) & $27(37)$ & $9(21)$ & \\
\hline M6, n (\%) & $6(8)$ & $3(7)$ & \\
\hline $\mathrm{M} 7, \mathrm{n}(\%)$ & $0(0)$ & $1(2)$ & \\
\hline Not determined & 1 & 0 & \\
\hline Cytogenetic risk ${ }^{\mathrm{a}}$ & & & 1.00 \\
\hline Low, n (\%) & $5(8)$ & $2(5)$ & \\
\hline Intermediate, $\mathrm{n}(\%)$ & $36(61)$ & $23(62)$ & \\
\hline High, n (\%) & $18(31)$ & $12(32)$ & \\
\hline Missing & 15 & 5 & \\
\hline Treatment & & & 0.42 \\
\hline Intensive, n (\%) & $71(96)$ & $37(88)$ & \\
\hline $\begin{array}{l}\text { Palliative/supportive } \\
\text { care, n (\%) }\end{array}$ & $3(4)$ & $5(12)$ & \\
\hline
\end{tabular}

${ }^{\mathrm{a}}$ Cytogenetic risk is defined as: low, $\mathrm{t}(8 ; 21), \mathrm{t}(16 ; 16)$ or $\operatorname{inv}(16)$; intermediate, normal karyotype or all other abnormalities not classified into low or high risk group; high, inv(3), t( $3 ; 3),-5$, $\operatorname{del}(5 q),-7, \operatorname{del}(7 q), 11 q 23$-aberrations, complex karyotype defined by 3 or more chromosomal aberrations.

Since AML of elderly patients display an increased incidence of chromosomal imbalances, we hypothesized that age-dependent differences in genes involved in spindle formation and chromosome segregation might be relevant. We therefore selected the two genes PRPF4 and SMC1A for further analysis of the protein expression as determined by immunohistochemistry of tissue microarrays.

PRPF4 is a kinetochore component necessary for a functional spindle assembly checkpoint (20). The spindle assembly checkpoint prevents the initiation of the anaphase by halting cells in M2 phase in the presence of chromosomes not attached to spindle apparatus microtubules (21). PRPF4 depletion by siRNA leads to chromosomal segregation defects and aneuploidy (20). However, the detection of PRPF4 protein expression was not informative in our study. 
A

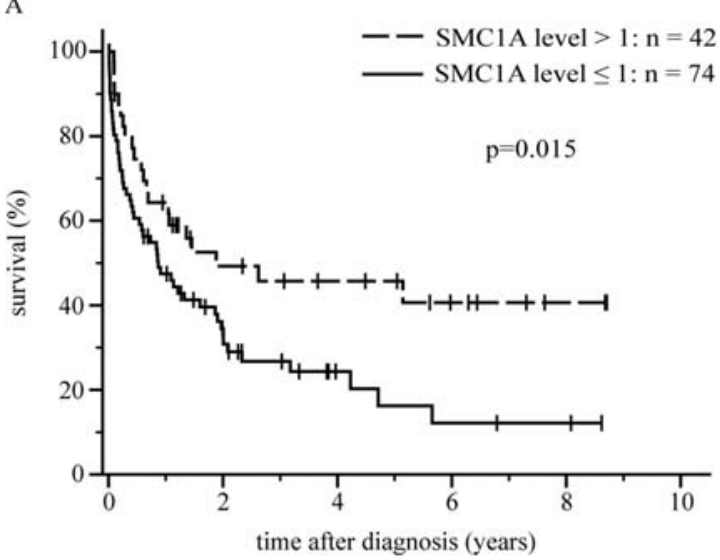

B

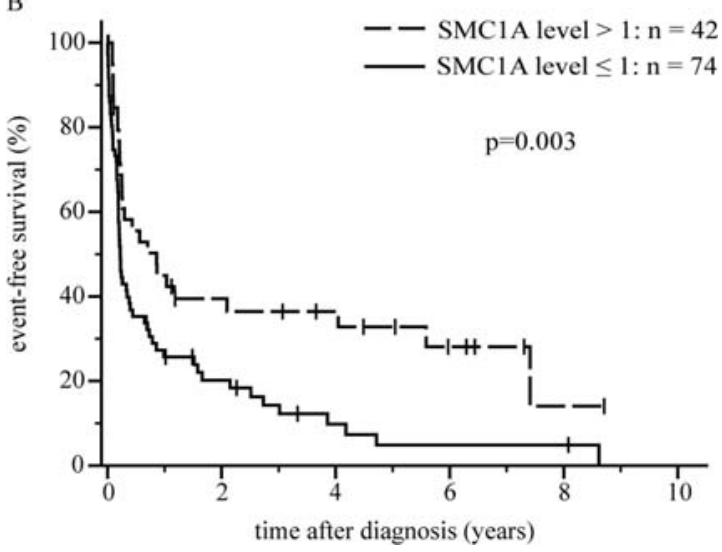

Figure 4. Association of SMC1A protein expression with event free and overall survival in AML patients. (A) Kaplan-Meier plot of the overall survival stratified by SMC1A protein expression. (B) Event free survival stratified by SMC1A protein expression.
On the other hand, low SMC1A protein levels were a strong predictor of impaired survival in AML. SMC1A belongs to the highly conserved structural maintenance of chromosome genes. The human SMC1A protein is part of the cohesin multiprotein complex which is required for sister chromatin cohesion during mitosis (22). SMC1A was found to be mutated in some colorectal cancers (23). In addition, one study demonstrated an upregulation of SMC1A mRNA in cervix cancer cells compared to normal cervix (24). No protein expression studies with SMC1A in cancer cells have been published to date. Recently, SMC1A was identified as an important gene for self-renewal in embryonic stem cells and a knock-down of SMC1A protein expression by siRNA results in an impaired self-renewal capacity of murine embryonic stem cells (25).

Hypothetically, a loss of SMC1A protein expression might lead to a chromosomal instability and aneuploidy due to the disruption of sister chromatin cohesion. However, no correlation was found between either SMC1A protein expression and the presence of a complex-aberrant karyotype or SMC1A protein expression and the presence of any cytogenetical abnormalities versus a normal karyotype in our study.

We did not observe a direct correlation between SMC1A mRNA and protein levels. The median age of patients with low SMC1A expression was higher than the age of patients with moderate to high SMC1A expression, but this difference was statistically not significant. These findings suggest complex regulation of SMC1A protein levels with mRNA levels being only one of them. It has been previously published that SMC1A mRNA expression detected by oligonucleotide microarray did not correlate with prognosis in a set of 285

Table III. Univariate (log-rank test) and multivariate Cox regression analysis.

\begin{tabular}{lcc}
\hline & $\begin{array}{c}\text { Event free survival } \\
\text { p-value }\end{array}$ & $\begin{array}{c}\text { Overall survival } \\
\text { p-value }\end{array}$ \\
\hline Univariate analysis & 0.030 \\
Age $(<60$ vs. $\geq 60$ years $)$ & 0.025 \\
Gender & 0.028 \\
De novo versus secondary leukemia, & 0.537 \\
Cytogenetic risk $($ low, intermediate, high) & 0.004 \\
LDH serum level $(<700$ vs. $\geq 700$ U/l) & 0.227 \\
White blood count $(<20,000$ vs. $\geq 20,000 / \mu 1)$ & 0.003 \\
SMC1A protein level $(\leq 1$ vs. $>1)$ & \\
Multivariate analysis & 0.003 \\
Age $(<60$ vs. $\geq 60$ years $)$ & 0.026 \\
Gender & 0.103 \\
De novo versus secondary leukemia & 0.123 \\
Cytogenetic risk $($ low, intermediate, high) & \\
LDH serum level $(<700$ vs. $\geq 700$ U/l) & 0.049 \\
White blood count $(<20,000$ vs. $\geq 20,000 / \mu 1)$ & 0.007 \\
SMC1A protein level $(\leq 1$ vs. $>1)$ & 0.014 \\
\hline
\end{tabular}

Known risk factors and SMC1A protein level were analyzed with regard to event free and overall survival. Variables with significant correlation in the univariate analysis were included in the multivariate analysis. 
AML samples (26). Regardless of the underlying mechanisms of regulation, the association of low SMC1A mRNA with aging and the poor prognosis of low SMC1A protein correspond well with each other.

mRNA expression level of certain genes have been identified as a prognostic marker in AML (4-6). However, given the lack of correlation with protein expression, few if any of the identified mRNAs might actually play a direct role in the mechanisms that lead to AML relapse. Thus, independent verification on the protein level is required before any conclusions on the biological relevance can be drawn. In addition, the expression of proteins in AML blasts at diagnosis, i.e. P-glycoprotein, is able to identify prognostic subgroups in AML. Tissue microarrays are a powerful tool to discover the protein expression level on a high-throughput level (27). Our approach was suitable to test a hypothesis regarding the protein expression level on a high number of samples. With this approach, we identified SMC1A protein expression as a potential new prognostic marker in AML which can be easily assessed on bone marrow biopsy samples obtained at diagnosis.

\section{Acknowledgements}

The authors are grateful to Ms. Christine Disselhoff, Ms. Judith Obernuefemann and Ms. Ulrike Neubert for their technical assistance.

\section{References}

1. Mrozek K, Heerema NA and Bloomfield CD: Cytogenetics in acute leukemia. Blood Rev 18: 115-136, 2004.

2. Schlenk RF, Dohner K, Krauter J, et al: Mutations and treatment outcome in cytogenetically normal acute myeloid leukemia. $\mathrm{N}$ Engl J Med 358: 1909-1918, 2008.

3. Paschka P, Marcucci G, Ruppert AS, et al: Wilms' tumor 1 gene mutations independently predict poor outcome in adults with cytogenetically normal acute myeloid leukemia: a cancer and leukemia group B study. J Clin Oncol 26: 4595-4602, 2008.

4. Marcucci G, Maharry K, Whitman SP, et al: High expression levels of the ETS-related gene, ERG, predict adverse outcome and improve molecular risk-based classification of cytogenetically normal acute myeloid leukemia: a Cancer and Leukemia Group B Study. J Clin Oncol 25: 3337-3343, 2007.

5. Baldus CD, Tanner SM, Ruppert AS, et al: BAALC expression predicts clinical outcome of de novo acute myeloid leukemia patients with normal cytogenetics: a Cancer and Leukemia Group B Study. Blood 102: 1613-1618, 2003.

6. Heuser M, Beutel G, Krauter J, Dohner K, von Neuhoff N, Schlegelberger B and Ganser A: High meningioma 1 (MN1) expression as a predictor for poor outcome in acute myeloid leukemia with normal cytogenetics. Blood 108: 3898-3905, 2006.

7. Barjesteh van Waalwijk van Doorn-Khosrovani S, Erpelinck C, van Putten WL, et al: High EVI1 expression predicts poor survival in acute myeloid leukemia: a study of 319 de novo AML patients. Blood 101: 837-845, 2003.

8. Campos L, Guyotat D, Archimbaud E, et al: Clinical significance of multidrug resistance P-glycoprotein expression on acute non-lymphoblastic leukemia cells at diagnosis. Blood 79: 473-476, 1992.

9. List AF, Spier CS, Grogan TM, et al: Overexpression of the major vault transporter protein lung-resistance protein predicts treatment outcome in acute myeloid leukemia. Blood 87: 2464-2469, 1996.
10. Paschka P, Marcucci G, Ruppert AS, et al: Adverse prognostic significance of KIT mutations in adult acute myeloid leukemia with inv(16) and $t(8 ; 21)$ : a Cancer and Leukemia Group B Study. J Clin Oncol 24: 3904-3911, 2006.

11. Schlenk RF, Benner A, Krauter J, et al: Individual patient databased meta-analysis of patients aged 16 to 60 years with core binding factor acute myeloid leukemia: a survey of the German Acute Myeloid Leukemia Intergroup. J Clin Oncol 22: 3741-3750, 2004.

12. Buchner T, Berdel WE, Schoch C, et al: Double induction containing either two courses or one course of high-dose cytarabine plus mitoxantrone and postremission therapy by either autologous stem-cell transplantation or by prolonged maintenance for acute myeloid leukemia. J Clin Oncol 24: 2480-2489, 2006.

13. Leith CP, Kopecky KJ, Godwin J, et al: Acute myeloid leukemia in the elderly: assessment of multidrug resistance (MDR1) and cytogenetics distinguishes biologic subgroups with remarkably distinct responses to standard chemotherapy. A Southwest Oncology Group study. Blood 89: 3323-3329, 1997.

14. Leith CP, Kopecky KJ, Chen IM, et al: Frequency and clinical significance of the expression of the multidrug resistance proteins MDR1/P-glycoprotein, MRP1, and LRP in acute myeloid leukemia: a Southwest Oncology Group Study. Blood 94: 1086$1099,1999$.

15. Buchner T, Berdel WE, Haferlach C, et al: Older age is an independent risk factor in AML. Blood 112: A555, 2008.

16. Buchner T, Berdel WE, Haferlach C, et al: Age-related risk profile and chemotherapy dose response in acute myeloid leukemia: a study by the German Acute Myeloid Leukemia Cooperative Group. J Clin Oncol 27: 61-69, 2009.

17. Schoch C, Kern W, Schnittger S, Buchner T, Hiddemann W and Haferlach T: The influence of age on prognosis of de novo acute myeloid leukemia differs according to cytogenetic subgroups. Haematologica 89: 1082-1090, 2004.

18. Wang Y, Barbacioru C, Hyland F, et al: Large scale real-time PCR validation on gene expression measurements from two commercial long-oligonucleotide microarrays. BMC Genomics 7: 59,2006

19. Kononen J, Bubendorf L, Kallioniemi A, et al: Tissue microarrays for high-throughput molecular profiling of tumor specimens. Nat Med 4: 844-847, 1998.

20. Montembault E, Dutertre S, Prigent C and Giet R: PRP4 is a spindle assembly checkpoint protein required for MPS1, MAD1, and MAD2 localization to the kinetochores. J Cell Biol 179: 601-609, 2007.

21. Vogt E, Kirsch-Volders M, Parry J and Eichenlaub-Ritter U: Spindle formation, chromosome segregation and the spindle checkpoint in mammalian oocytes and susceptibility to meiotic error. Mutat Res 651: 14-29, 2008.

22. Strunnikov AV and Jessberger R: Structural maintenance of chromosomes (SMC) proteins: conserved molecular properties for multiple biological functions. Eur J Biochem 263: 6-13, 1999.

23. Barber TD, McManus K, Yuen KW, et al: Chromatid cohesion defects may underlie chromosome instability in human colorectal cancers. Proc Natl Acad Sci USA 105: 3443-3448, 2008.

24. Narayan G, Bourdon V, Chaganti S, et al: Gene dosage alterations revealed by cDNA microarray analysis in cervical cancer: identification of candidate amplified and overexpressed genes. Genes Chromosomes Cancer 46: 373-384, 2007.

25. Hu G, Kim J, Xu Q, Leng Y, Orkin SH and Elledge SJ: A genome-wide RNAi screen identifies a new transcriptional module required for self-renewal. Genes Dev 23: 837-848, 2009.

26. Valk PJ, Verhaak RG, Beijen MA, et al: Prognostically useful gene-expression profiles in acute myeloid leukemia. N Engl J Med 350: 1617-1628, 2004.

27. Bubendorf L, Nocito A, Moch H and Sauter G: Tissue microarray (TMA) technology: miniaturized pathology archives for high-throughput in situ studies. J Pathol 195: 72-79, 2001. 